\title{
Bullying and Victimization, Depressive Mood, and Non-Suicidal Self-Injury in Adolescents: The Moderating Role of Parental Support
}

\author{
Laurence Claes $\cdot$ Koen Luyckx $\cdot$ Imke Baetens • \\ Monique Van de Ven · Cilia Witteman
}

(C) Springer Science+Business Media New York 2015

\begin{abstract}
We investigated the associations of bullying and victimization with non-suicidal self-injury (NSSI), as well as the mediating role of depressive mood in a sample of 785 adolescents. Further, we explored the moderating role of parental support in these associations. All participants completed questionnaires on bullying, victimization, NSSI, depressive mood, and parental support. Almost $21 \%$ of adolescents engaged in at least one form of NSSI. Both bullying and victimization of being bullied increased the risk of engaging in NSSI. The relationships of both bullying and victimization with NSSI were partially mediated by depressive mood. Finally, the relationships of both bullying and victimization with NSSI and of depressive mood with NSSI were found to be moderated by parental support, indicating that these relationships were less pronounced in adolescents who experienced high parental support. Hence, we underscore the need to include both emotion-regulation training and family-based interventions into bully intervention programs to prevent NSSI.
\end{abstract}

Keywords Bullying · Victimization · Non-suicidal self-injury $\cdot$ Depressive mood $\cdot$ Parental support

L. Claes $(\bowtie) \cdot$ K. Luyckx $\cdot$ I. Baetens

Faculty of Psychology and Educational Sciences, Katholieke Universiteit Leuven, Tiensestraat 102, 3000 Louvain, Belgium e-mail: Laurence.Claes@ppw.kuleuven.be

M. Van de Ven · C. Witteman

Behavioral Science Institute, Diagnostic Decision Making,

Radboud Universiteit Nijmegen, Nijmegen, The Netherlands

\section{Introduction}

Non-suicidal self-injury (NSSI) refers to the deliberate and direct injury of one's own body tissue without suicidal intent, such as scratching, cutting, hitting, and burning oneself (Claes and Vandereycken 2007; Nock and Favazza 2009). NSSI is a pervasive public health problem, and adolescence seems to be a period of increased risk for NSSI (Nock and Prinstein 2004). Muehlenkamp et al. (2012) found an average lifetime estimate of $18 \%$ for NSSI (i.e., at least one episode of NSSI) in adolescent samples across the globe. When using the DSM-5 criteria for a potential NSSI diagnosis, the average life-time prevalence of NSSI in adolescents was estimated around $6.7 \%$ (Zetterqvist et al. 2013). Results from studies on gender differences in NSSI in youth have been mixed, with some studies suggesting that girls engage in NSSI more often than boys (e.g., Guerry and Prinstein 2010) and other studies showing no gender differences (Hilt et al. 2008).

Only a few studies have investigated the association between being a victim of bullying and NSSI (Noble et al. 2011). Being a victim of bullying - also called bullying victimization-refers to being a victim of "aggressive behavior or intentional harm-doing, which is performed repeatedly over time, and which involves an imbalance in power" (Luukkonen et al. 2009, p. 131). The association between bullying victimization and NSSI can be mediated by variables such as self-derogation, self-criticism and low self-esteem. A majority of individuals who self-injure report reasons such as "to punish myself" or "to express anger at self/others" (Klonsky 2007, 2009). Further, although a handful of studies has investigated the association between victimization and NSSI, even fewer studies focused on the association between bullying itself and NSSI (e.g., Barker et al. 2008). Therefore, our study adds 
to the existing literature by exploratory investigating the association between bullying and NSSI, besides bullying victimization and NSSI.

Bullying and victimization peak in prevalence and frequency in early adolescence (Scholte et al. 2013), and most adolescents follow a declining trajectory of bullying and victimization from early to mid-adolescence (Barker et al. 2008). With respect to the prevalence, boys more often tend to be bullies and girls more often victims of bullying (Klomek et al. 2007; Luukkonen et al. 2009). Whereas victimization is related to emotional adjustment problems such as loneliness, anxiety, low self-esteem, and depression (Eslea et al. 2003; Hawker and Boulton 2000; Kochenderfer-Ladd and Wardrop 2001, Luukkonen et al. 2009; Nansel et al. 2001), bullies are at increased risk for aggressive and delinquent behaviors, school failure, and dropping out (Barker et al. 2008) on the one hand, but also at increased risk for depression, suicide ideation, and suicide attempts on the other hand (e.g., Klomek et al. 2007; Seals and Young 2003).

A handful of cross-sectional studies has shown that male and female adolescents who reported being the victim of bullying were at an increased risk for NSSI. O'Conner and colleagues (2009) found that boys who reported being bullied in school were twice as likely to engage in selfharming behaviors (including both suicidal and NSSI behaviors). Girls who reported being bullied in school were three times as likely to engage in these self-harming behaviors. Hay and Meldrum (2010) also showed that bullying victimization was significantly and positively associated with NSSI. More recently, Bakken and Gunter (2012) and Alfonso and Kaur (2012) also found that individuals who reported high levels of victimization were more likely to engage in self-injury with or without suicidal intent than those who reported low levels of victimization. Additionally, bullies showed an increased risk to engage in suicidal as well as NSSI behaviors, certainly when they had a history of being bullied, the so called bully-victims (Barker et al. 2008).

Additionally, Hay and Meldrum (2010) showed that the relationship between victimization and NSSI was partially mediated by negative emotions. Victimization increases the level of psychological distress, which, in turn, increases the risk to engage in NSSI (see Clements-Nolle et al. 2006; Diaz et al. 2001). Many studies have found a positive relationship between victimization and depressive symptoms (for a review, see Hawker and Boulton 2000), which can be explained by stress theory (Zautra 2003). Victimization and the inability to react adequately to such victimization can cause stress, which, in turn, affects psychosocial functioning and causes emotions such as fear, depression, and anger (Pouwelse et al. 2011). Subsequently, these negative emotions can trigger self-harming behaviors such as NSSI to cope with these negative emotions. Several studies have indeed shown the affect-regulation function of NSSI (e.g., decreasing negative emotions) as well as the self-punishment function (driven by anger) of these behaviors (e.g., Claes et al. 2010; Klonsky 2007, 2009).

Finally, Hay and Meldrum (2010) have shown that the relationship between bullying victimization and NSSI was highly conditional, that is, the association between victimization and NSSI almost disappeared for adolescents who were exposed to supportive parenting practices. Additionally, other studies have also shown that parental support may play a buffering role between victimization and internalizing distress caused by bullying (e.g., Davidson and Demaray 2007; Flaspohler et al. 2009; Flouri and Buchanan 2002), and between psychological distress and NSSI (e.g., Baetens et al. 2014; Tatnell et al. in press). These findings are in line with the buffering hypothesis of social support (Cohen and Wills 1985; Jackson 1992) which states that social support can function as a buffer against different types of stress (Pouwelse et al. 2011; Wolff et al. 2014).

The aim of the present study was partially to replicate findings of previous studies linking bullying victimization to NSSI, and to add to the existing knowledge by also investigating the association between being a bully and NSSI. Additionally, we take into account the mediating role of depressive mood and the moderating role of parental support in the relationship of bullying and victimization with NSSI. Therefore, the present study had three main research goals. First, we investigated the extent to which bullying and victimization were related to NSSI. Second, we investigated whether depressive mood mediated the relationship of bullying and victimization with NSSI. Finally, we investigated whether the relation between bullying/victimization, depressive mood, and NSSI was moderated by the level of perceived parental support by the adolescent. Based on previous studies (e.g., Barker et al. 2008; Hay and Meldrum 2010), we hypothesized a positive association between being a victim of bullying or being a bully and NSSI. Additionally, according to the stress theory of Zautra (2003), we hypothesized that the relationships of bullying and victimization with NSSI would be partially mediated by depression, given that both bullying and victimization are related to depressive symptoms (e.g., Barker et al. 2008), and depressive symptoms are strongly related to NSSI (Nock et al. 2006; Whitlock et al. 2006). Finally, and based on the buffering hypothesis of social support (Cohen and Wills 1985; Jackson 1992), we hypothesized that the association between bullying victimization and NSSI would be moderated by parental support (Hay and Meldrum 2010). Additionally, parental support could moderate the 
association between victimization and depressive mood given that parental support could temper the stress caused by the victimization (e.g., Davidson and Demaray 2007). In keeping with the idea that parent support may decrease negative affect, we also hypothesized that parents support would mediate the association between depressive mood and NSSI (e.g., Baetens et al. 2014). At an exploratory level, we also investigated whether the association between bullying, depressive mood, and NSSI would be moderated by parental support. In this way, we wanted to test an integrative model in which the components being a bully/ being bullied, depressive mood, parental support and NSSI are integrated.

\section{Method}

\section{Participants}

The convenience sample consisted of 1,013 high school students (7-12th grade). After eliminating 204 students who had missing data on the variables under study and after eliminating 24 univariate and multivariate outliers on the maternal/paternal support variables, the remaining sample consisted of 785 students. Of these 785 high school students, $349(44.5 \%)$ were female and $436(55.5 \%)$ were male. Mean age was 15.56 years $(S D=1.32$; range 12-19).

\section{Procedure}

Several high schools, located in different areas of Belgium and the Netherlands, participated in the study. All students who were present the day of the study completed questionnaires during regular school hours. Students were provided with an envelope including the questionnaires and for the Belgian students an informed consent form. Students from the Netherlands did not receive an informed consent form, given that in the Netherlands no written informed consent form was necessary to invite students to participate in anonymous questionnaire research. After completing the forms, they returned them in a sealed envelope to the teacher or the researcher, who remained in the classrooms while the students completed the questionnaires. The study was approved by the ethical board of both universities. Participants were not compensated for participating in the study.

Measures

\section{Bullying and Victimization}

To assess involvement in bullying and victimization, we used the Bully/Victim Self-Report Questionnaire (Olweus
1991), of which five items assess being a victim of direct bullying ( $\alpha=.78$ in the present study, e.g., How often were you left out of things, excluded or ignored?); and the other five items assess how much a person actively participated in the bullying of others $(\alpha=.66$, e.g., How often do you spread rumors about other students?). All items were scored on a 5-point Likert scale ranging from 'never' to 'different times a week'.

To assess the presence of different types of NSSI behaviors, adolescents completed the Self-Harm Inventory (SHI; Sansone et al. 1998), which consists of 22 yes/no items inquiring whether the participant had ever intentionally engaged in the specified behavior (e.g., cutting). In the present study only the NSSI subscale (cutting, burning, hitting, scratching and head-banging) was used and we added that these behaviors needed to be engaged in without suicidal intent, with a Cronbach's alpha of .66. Besides the five different types of NSSI behaviors, adolescents also indicated the age of onset of NSSI. Adolescents who engaged in at least one form of NSSI was classified in the category 'NSSI'; whereas those without NSSI belonged to the category 'no NSSI'. In all analyses, the presence/ absence of NSSI (0/1) was used as dependent variable.

\section{Depressive Mood Symptoms}

The assess the extent to which adolescents experienced negative mood, the 6-item Depressive Mood List was used (Kandel and Davies 1986; Dutch translation: De Vries et al. 2003). Respondents were asked to report the frequency of experiences of negative feelings during the last 12 months. Each item was rated on a 5-point Likert scale ranging from 'never' to 'always'. Cronbach's alpha was .79.

\section{Perceived Parental Support}

To assess parental support, participants completed a 12-item version of the Relational Support Inventory (RSI; Scholte et al. 2001). The scale measures aspects of emotional and instrumental support by fathers and mothers separately. Sample items are: 'My mother/father supports me in the things I do', and 'My mother/father explains or shows how I can make or do something'. Each item was rated on a 5-point Likert scale ranging from 'very untrue' to 'very true'. Cronbach's alpha was .85 for maternal support, and .86 for paternal support.

\section{Data Analyses}

To calculate the associations between the presence/absence of NSSI and categorical variables, we used the Chi Square test statistic. To investigate whether adolescents differed with respect to victimization, bullying, depressive mood, 
and parental support, we performed a MANOVA with the presence/absence of NSSI as independent variable, and the aforementioned variables as dependent variables.

To test for mediation of depressive mood between bully victimization and NSSI, three path models were estimated (Holmbeck 1997): (a) a direct effect model including victimization and NSSI; (b) a full mediation model in which victimization is indirectly related to NSSI through depressive mood; and (c) a partial mediation model including both direct paths from victimization to NSSI and indirect paths through depressive mood. The same procedure was repeated to test for mediation of depressive mood between bullying and NSSI. Full mediation is demonstrated when the addition of the direct paths does not improve model fit. To evaluate model fit, we used the Chi squared index, which should be as small as possible; the Root Mean Square Error of Approximation (RMSEA), which should be less than .10, and preferably .06; and the Comparative Fit Index (CFI), which should exceed .90, and preferably .95 (Hu and Bentler 1999; Kline 2005). To compare model fit between the full and partial mediation models, the more parsimonious full mediation model was favoured if $\Delta \chi^{2}$ was not significant at $p<.05$ and if $\Delta \mathrm{CFI}$ was equal to or smaller than .01 (Cheung and Rensvold 2002; Vandenberg and Lance 2000). As such, omitting the direct path from victimization/bullying to NSSI in the full mediation model would not be accompanied by a significant decrease in model fit for both these indices as compared to the partial mediation model. The BOOTSTRAP option available in Mplus 6.0 (number of bootstrap draws set to 1,000) was used. Preliminary correlational analyses indicated that gender was positively related to depressive $\operatorname{mood}(r=.28, p<.001)$ and negatively to bullying $(r=-.13, p<.001)$, and age was positively related to depressive mood $(r=.12, p<.01)$ and negatively to bully victimization $(r=-.16, p<.001)$. Hence, these four associations were controlled for in the respective path models.

Using multi-group path analyses we examined whether the directional paths obtained in the direct effects and partial mediation models would be moderated by parental support. The null hypothesis of invariant path coefficients across parental support groups would be rejected if at least one of the following criteria was satisfied: $\Delta \chi^{2}$ significant at $p<.05$; or $\Delta \mathrm{CFI}>.01$, indicating that constraining path coefficients would be accompanied by a significant decrease in one of these fit indices. If both criteria were not satisfied (i.e., $\Delta \chi^{2}$ not significant at $p<.05$ and $\Delta \mathrm{CFI}$ equal to or smaller than .01), the more parsimonious invariant model was favored. Prior to conducting these analyses, a cluster-analysis was conducted using a two-step procedure (Gore 2000) to identify individuals scoring high versus low on perceived paternal and maternal support. In the first step, after removing 24 univariate and multivariate outliers on the parental support variables, a hierarchical cluster analysis was carried out on standardized paternal and maternal support scores using Ward's method based on squared Euclidian distances. In the second step, these initial cluster centres were used as non-random starting points in an iterative $k$-means clustering procedure. The twocluster solution explained 52 and $56 \%$ of the variance in paternal and maternal support, respectively, and, hence, was considered adequate in terms of variance explained. The first cluster $(N=410)$ scored relatively low on paternal $(z=-0.63)$ and maternal support $(z=-0.59)$, and the second cluster $(N=375)$ scored relatively high on paternal $(z=0.77)$ and maternal support $(z=0.74)$ (with all $z$-scores reflecting moderate effects; Cohen 1988).

\section{Results}

Overall, $20.1 \%(N=158)$ of the 785 adolescents engaged in at least one form of NSSI, with no significant difference between boys and girls $\left[\chi^{2}(1)=.02, n s\right]$, and with no significant relationship with age $(r=-0.03, n s)$. Twelve percent $(N=94)$ of the adolescents engaged in head banging, $8.2 \%(N=64)$ scratching oneself, $8 \%(N=63)$ hitting oneself, $5.6 \%(N=44)$ cutting oneself, and $1.9 \%$ ( $N=15$ ) burning oneself. The mean age of onset of NSSI was 12.43 years $(S D=2.80$, range $2-17$ years).

The mean level of victimization was $1.33(S D=0.48$, range 1-5), and the mean level of bullying was 1.33 $(\mathrm{SD}=0.38$, range $1-4)$. We did not find a significant gender difference in victimization $[F(1,783)=3.38, n s]$; however, boys $(M=1.38, S D=.43)$ more often reported bullying their peers than girls $(M=1.28, S D=.31) \quad[F(1$, $783)=12.49, p<.001]$. Additionally, being a victim of bullying was significantly negatively related to age $(r=$ $-0.16, p<0.001)$, whereas being a bully was not $(r=$ $-0.01, \mathrm{~ns})$. The correlation between victimization and bullying was $r=.27(p<.001)$.

Concerning research aim 1, adolescents with NSSI scored significantly higher on victimization, bullying, and depressive mood compared to adolescents without NSSI. On the contrary, adolescents with NSSI experienced less parental support than adolescents without NSSI (see Table 1).

According to the second research aim, we tested for mediation of depressive mood between bully (victimization) and NSSI. For bullying victimization, the direct effects model provided an adequate fit to the data $\left(\chi^{2}(1)=0.03\right.$, $p=.86$; CFI $=1.000$; RMSEA $=.000)$. NSSI was positively predicted $(\beta=.23, p<.001)$ by being a victim of bullying. Next, the full mediation model had a less than adequate fit $\left(\chi^{2}(5)=46.40, p<.001 ; \quad \mathrm{CFI}=.826\right.$; RMSEA $=.103)$, whereas the partial mediation model 
Table 1 Means (standard deviations) on victimization, bullying, depressive mood and parental support (aggregated over father and mother) of adolescents with and without NSSI $(N=785)$

\begin{tabular}{|c|c|c|c|c|c|}
\hline & \multicolumn{2}{|c|}{ No NSSI $(N=627)$} & \multicolumn{2}{|c|}{ NSSI $(N=158)$} & \multirow[t]{2}{*}{$F(1,783)$} \\
\hline & $M$ & $(S D)$ & $M$ & $(S D)$ & \\
\hline Victim & 1.27 & $(0.42)$ & 1.55 & $(0.63)$ & $43.81 * * *$ \\
\hline Bully & 1.31 & $(0.36)$ & 1.44 & $(0.46)$ & $14.75 * * *$ \\
\hline Depressive mood & 2.49 & $(0.69)$ & 2.90 & $(0.70)$ & $44.91 * * *$ \\
\hline Parental support & 3.97 & $(0.51)$ & 3.66 & $(0.51)$ & $48.60 * * *$ \\
\hline
\end{tabular}

$* * * p<.001$

$\left(\chi^{2}(4)=16.44, p=.0 .003 ; \mathrm{CFI}=.948 ; \mathrm{RMSEA}=.063\right)$ had a significantly better fit $\left(\Delta \chi^{2}(1)=29.96, p<.001\right.$; $\Delta \mathrm{CFI}$ in favor of the partial mediation model). Figure 1 presents the partial mediation model accounting for $9.2 \%$ of the variance in NSSI. The indirect effect of being a victim on NSSI via depressive mood was significant [point estimate $=.035$, S.E. $=.009,95 \%$ CI $(.017, .054)]$.

For being a bully, the direct effects model provided an adequate fit to the data $\left(\chi^{2}(1)=0.39, p=.53\right.$; $\mathrm{CFI}=1.000$; RMSEA $=.000)$. NSSI was positively predicted $(\beta=.14, p<.001)$ by 'being a bully'. Next, the full mediation model had a less than adequate fit, especially in terms of CFI $\left(\chi^{2}(5)=23.56, p<.001 ; \mathrm{CFI}=.897\right.$; RMSEA $=.069)$, whereas the partial mediation model $\left(\chi^{2}\right.$ $(4)=14.40, p=.0 .006 ; \quad$ CFI $=.943 ;$ RMSEA $=.058)$ had a significantly better fit $\left(\Delta \chi^{2}(1)=9.16, p=.003\right.$; $\Delta \mathrm{CFI}$ in favor of the partial mediation model). Figure 2 presents the partial mediation model accounting for $6.6 \%$ of the variance in NSSI. The indirect effect from bullying to NSSI via depressive mood was significant (point estimate $=.032$, S.E. $=.010,95 \%$ CI $[.012, .052])$.

According to the third research aim, we examined the moderating role of parenting clusters on the previously obtained direct effects models. For victimization, the constrained model (in which the path from victimization to NSSI was fixed as equal in both parenting clusters) had a significantly worse fit than the model in which this path was freely estimated in both parenting clusters $\left(\Delta \chi^{2}(1)=4.10\right.$, $p=.04 ; \Delta \mathrm{CFI}=.028)$. Hence, the unconstrained model was retained (low parental support: $\beta=.24, p<.001$; high parental support: $\beta=.13, p=.05$ ). For bullying, the unconstrained model was again favored over the constrained model $\left(\Delta \chi^{2}(1)=4.41, p=.04 ; \Delta \mathrm{CFI}=.153\right)$, pointing to the fact that the direct path from bullying to NSSI was again moderated by parental support (low parental support: $\beta=.16, p<.01$; high parental support: $\beta=.03, p=.65$ ).

Finally, we examined the moderating role of the parenting clusters on the partial mediation models. For victimization, invariance tests indicated that constraining all three paths (i.e., from victimization to NSSI and to depressive mood and from depressive mood to NSSI) as equal for both parenting clusters was accompanied by a significantly worse fit in terms of $\Delta \mathrm{CFI}=.019$ and marginally so in terms of $\Delta \chi^{2}(3)=7.27, p=.06$. Figure 3 presents all freely estimated standardized path coefficients. Ancillary analyses demonstrated that especially the path from victimization to NSSI could not be unequivocally constrained as equal: although $\Delta \chi^{2}(1)=3.31, p=.07$ was not significant, $\Delta \mathrm{CFI}=.011$ pointed to a decrease in fit. For bullying, invariance tests indicated that constraining all three paths as equal for both parenting clusters again was accompanied by a significantly worse fit in terms of $\Delta \mathrm{CFI}=.029$ and $\Delta \chi^{2}(3)=8.14, p=.04$. Figure 4 presents all freely estimated standardized path coefficients. Ancillary analyses demonstrated that especially the path from bullying to NSSI $\left(\Delta \mathrm{CFI}=.017\right.$ and $\Delta \chi^{2}(1)=3.99$,

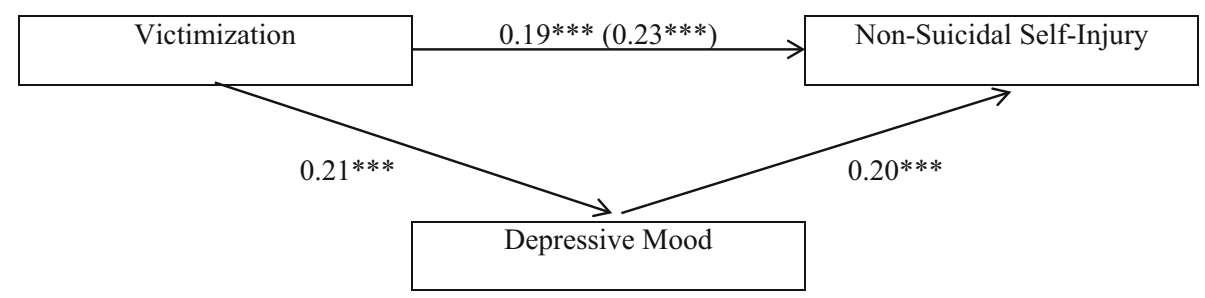

Fig. 1 Final partial mediation model in the total sample. Associations with gender and age are not shown. All coefficients are standardized. Coefficients between parentheses represent the coefficients from the direct effects model. $* p<.05 . * * p<.01 . * * * p<.001$ 


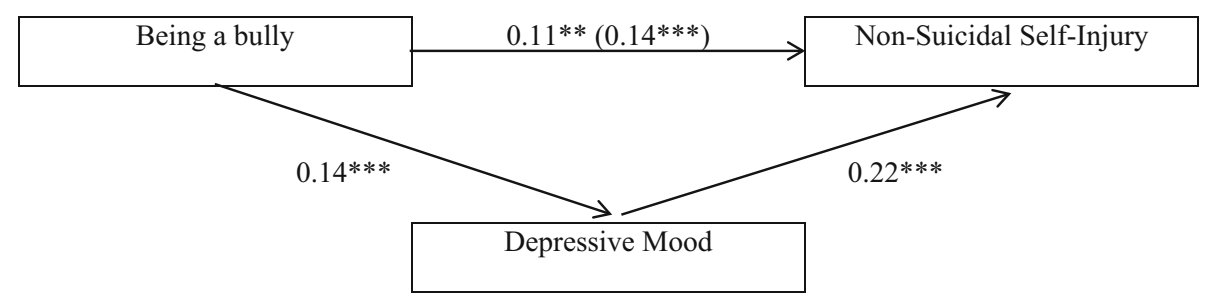

Fig. 2 Final partial mediation model in the total sample. Associations with gender and age are not shown. All coefficients are standardized. Coefficients between parentheses represent the coefficients from the direct effects model. $* p<.05 . * * p<.01 . * * * p<.001$

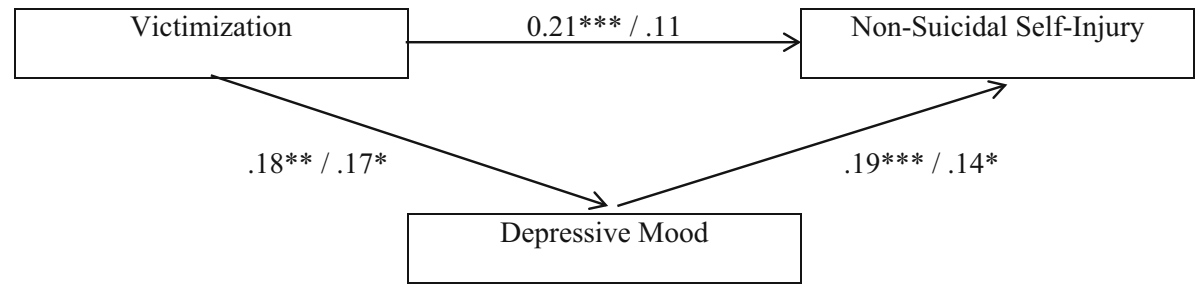

Fig. 3 Unconstrained partial mediation model. Associations with gender and age are not shown. All coefficients are standardized. Coefficients before the slash are for low parental support, coefficients after the slash are for high parental support. *p $<.05$. **p $<.01$. ***p $p .001$

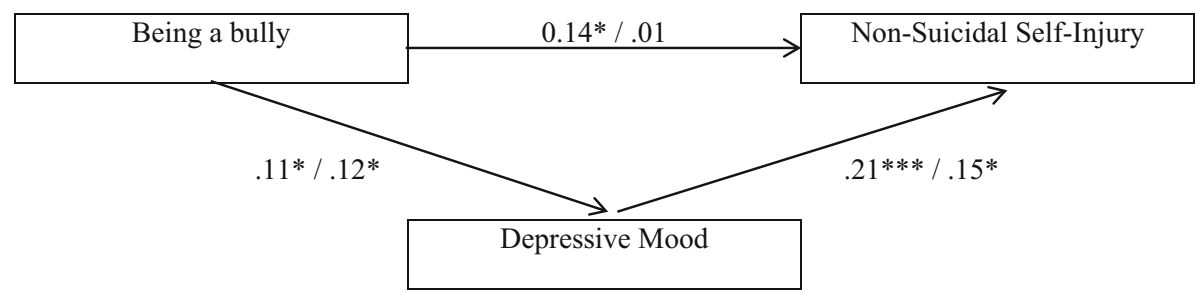

Fig. 4 Unconstrained partial mediation model. Associations with gender and age are not shown. All coefficients are standardized. Coefficients before the slash are for low parental support, coefficients after the slash are for high parental support. * $p<.05$. ${ }^{* *} p<.01$. $* * * p<.001$

$p=.04)$ and from depression to NSSI $\left(\Delta \chi^{2}(1)=3.14\right.$, $p=.08$ was not significant but $\Delta \mathrm{CFI}=.012$ pointed to a decrease in fit) could not be constrained as equal. Hence, both Figs. 3 and 4 show that parental support moderates the direct association between bullying/victimization and NSSI: this association becomes non-significant for adolescents who perceive their parents as supportive, and this is not the case for adolescents who perceive their parents as less supportive. A similar effect was found for the relationship between depressive mood and NSSI but only in the model for bullying (and not for victimization). Adolescents engaging in bullying who perceived their parents as supportive showed a weaker association between depressive mood and NSSI than adolescents engaging in bullying who perceived their parents as less supportive. Finally, the associations of bullying and victimization with depressive mood were not moderated by parental support in either model.

\section{Discussion}

In the present study, we investigated the association between bullying and victimization and NSSI in a sample of Flemish and Dutch adolescents. More specifically, we tested if this association was mediated by depressive mood and moderated by parental support, hereby re-testing and extending the model of Hay and Meldrum (2010). Overall, $20.1 \%$ of our sample of adolescents had ever engaged in at least one form of NSSI, and the rate of NSSI was similar for boys and girls, confirming results from previous studies (e.g., Muehlenkamp et al. 2011; Nock and Prinstein 2004). Adolescents with NSSI scored significantly higher on bullying victimization and bullying compared to adolescents without NSSI, confirming the findings of other studies (e.g., Barker et al. 2008; Hay and Meldrum 2010).

In line with previous studies (e.g., Diaz et al. 2001; Clements-Nolle et al. 2006; Hay and Meldrum 2010), we 
found that the association between being a victim of bullying and NSSI was partially mediated by depressive mood. According to Cassidy and Taylor (2005), the influence of victimization on mental health can be explained by stress theory (Zautra 2003, in Pouwelse et al. 2011). Frequent victimization and the inability to react adequately to this victimization can cause stress, fear, depression, and anger (Pouwelse et al. 2011), which can subsequently trigger self-harming behaviors (such as NSSI) to cope with these emotions. Several studies have already shown that affect-regulation and self-punishment are the two most common functions of NSSI (Klonsky 2007, 2009). We added to the existing literature by showing that the association between bullying and NSSI was also partially mediated by depressive mood. Both groups (victims as well as bullies) seem to be helped by developing more adequate emotion regulation skills to deal with their emotions (see also Noble et al. 2011).

Apart from the mediating role of depression, we also focused on a potential protective factor that could prevent adolescents from engaging in NSSI, even when being bullied or being a bully. The "buffering hypothesis of social support" (Cohen and Wills 1985; Jackson 1992 in Pouwelse et al. 2011; Wolff et al. 2014) argues that social support can function as a buffer against stress. In line with this buffering hypothesis, our results showed a moderating effect of perceived parental support on the associations between both bullying and victimization and NSSI. This means that the association of bullying and victimization with NSSI significantly decreased if adolescents perceived their parents as supportive, whereas this was not the case for adolescents who perceived their parents as less supportive. Hence, high levels of parental support 'buffered' the associations between both bullying and victimization and NSSI. Similar findings (but less pronounced) were found for the association between depressive mood and NSSI; adolescents who perceived their parents as more supportive showed less strong associations between depressive mood and NSSI as compared to adolescents who perceived their parents as less supportive. Hypothetically, parent support offered the adolescent the opportunity to cope with his/her depressive mood adequately instead of relying on NSSI. However, in contrast to previous studies, the associations between bullying and victimization and depressive mood did not seem to be moderated by parental support (e.g., Davidson and Demaray 2007; Flaspohler et al. 2009; Flouri and Buchanan 2002).

Based on these findings we want to formulate some clinical implications when developing interventions programs which focus on bully (victimization) and its unfavorable mental health consequences. First, it is important to include modules which focus both on victims of bullying as well as on bullies. Second, it also seems necessary to teach both victims and bullies how to adequately deal with depressive mood, which seems related with these adverse circumstances. They have to learn adequate emotion-regulation skills to cope with depressive feelings to prevent them from acting-out by means of NSSI (see e.g., Noble et al. 2011). Finally, it is may also be helpful to include family processes (i.e., family communication and involvement) as a possible intervention target in future bullying prevention efforts (e.g., Spriggs et al. 2007); as these factors seem to protect both victims and bullies from engaging in NSSI to regulate their emotions.

These findings and their implications should be viewed in the context of the study limitations: First, we only assessed the presence/absence of NSSI in adolescents. Therefore, future studies should also include other characteristics of NSSI, such as its' frequency and recency which could influence our findings. Secondly, all constructs were assessed through adolescent self-report only and the internal consistency of several measures was relatively low $(<.70)$. Although it is appropriate to gather information about subjective processes such as 'depressive mood' from the adolescents themselves, the use a single informant may have caused problems of shared method variance and selfpresentation bias (Smits et al. 2008). Therefore, it is important to replicate our findings with multi-informant assessment, certainly in the domain of bullying and parental behavior. (Crothers and Levinson 2004), for example, advise to assess bullying by multiple informants and by means of multiple assessment methods in different contexts over a longer period of time. Thirdly, our analyses were based on cross-sectional data gathered at one point in time, which prevent us from determining the causal order of the events. Future studies should therefore collect longitudinal data at different time points, to investigate the directionality of the relationships between bully (victimization), depressive mood, parental support, and NSSI. In the present model, we suggested that depressive mood triggered the NSSI behavior, whereas the opposite could also be the case. Furthermore, the collection of data at different time points would also make it possible the estimate developmental pathways from bullying (victimization) to NSSI or vice versa. Finally, the models accounted for a rather limited variance in NSSI (6-9 \%). Future studies should include additional variables in the models to increase the variance explained in NSSI (e.g., emotionregulation processes).

Despite these limitations, this study provides new insight in the relationship between bully (victimization), depressive mood, and NSSI, and the moderating (protective) role of parental support. 


\section{References}

Alfonso, M. L., \& Kaur, R. (2012). Self-injury among early adolescents: Identifying segments protected and at risk. Journal of School Health, 82, 537-547.

Baetens, I., Claes, L., Onghena, P., Grietens, H., Van Leeuwen, K., Pieters, C., et al. (2014). Is non-suicidal self-injury associated with parenting and family factors? Journal of Early Adolescence, 34, 387-405.

Bakken, N. W., \& Gunter, W. D. (2012). Self-cutting and suicidal ideation among adolescents: Gender differences in the causes and correlates of self-injury. Deviant Behavior, 33, 339-356.

Barker, E. D., Arseneault, L., Brendgen, M., Fontaine, N., \& Maughan, B. (2008). Joint development of bullying and victimization in adolescence: Relations to delinquency and self-harm. Journal of the American Academy of Child and Adolescent Psychiatry, 47, 9.

Cassidy, T., \& Taylor, L. (2005). Coping and psychological distress as a function of the bully victim dichotomy in older children. Social Psychology of Education, 8, 249-262.

Cheung, G. W., \& Rensvold, R. B. (2002). Evaluating goodness-of-fit indexes for testing measurement invariance. Structural Equation Modeling, 9, 233-255.

Claes, L., Klonsky, E. D., Muehlenkamp, W., Kuppens, P., \& Vandereycken, W. (2010). The affect-regulation function of nonsuicidal self-injury in eating- disordered patients: which affect states are regulated? Comprehensive Psychiatry, 51, 386-392.

Claes, L., \& Vandereycken, W. (2007). Self-injurious behaviour: Differential diagnosis and functional differentiation. Comprehensive Psychiatry, 48, 137-144.

Clements-Nolle, K., Marx, R., \& Katz, M. (2006). Attempted suicide among transgender persons. Journal of Homosexuality, 51, 53-69.

Cohen, J. (1988). Statistical power analysis for the behavioral sciences (2nd ed.). Hillsdale: Erlbaum.

Cohen, S., \& Wills, T. A. (1985). Stress, social support and the buffering hypothesis. Psychological Bulletin, 98, 310-357.

Crothers, L. M., \& Levinson, E. M. (2004). Assessment of bullying: A review of methods and instruments. Journal of Counseling and Development, 82, 496-503.

Davidson, L. M., \& Demaray, M. K. (2007). Social support as a moderator between victimization and internalizing-externalizing distress from bullying. School Psychology Review, 36, 383-405.

De Vries, H., Engels, R., Kremers, S., Wetzels, J., \& Mudde, A. (2003). Parents' and friends' smoking status as predictors of smoking onset: findings from six European countries. Health Education Research, 18, 627-636.

Diaz, R. M., Ayala, G., Bein, E., Henne, J., \& Marin, B. (2001). The impact of homophobia, poverty, and racism on the mental health of gay and bisexual Latino men: Findings from 3 US Cities. American Journal of Public Health, 91, 927-932.

Eslea, M., Menesini, E., Morita, Y., O’Moore, M., Mora-Merchan, J. A., Pereira, B., et al. (2003). Friendship and loneliness among bullies and victims: Data from seven countries. Aggressive Behavior, 30, 71-83.

Flaspohler, P. D., Elfstrom, J. L., Vanderzee, K. L., Sink, H. E., \& Birchmeier, Z. (2009). Stand by me: The effects of peer and teacher support in mitigating the impact of bullying on quality of life. Psychology in the Schools, 46, 636-649.

Flouri, E., \& Buchanan, A. (2002). Life satisfaction in teenage boys: The moderating role of father involvement and bullying. Aggressive Behavior, 28, 126-133.

Gore, P. A, Jr. (2000). Cluster analysis. In H. E. Q. Tinsley \& S. D. Brown (Eds.), Handbook of applied multivariate statistics and mathematical modeling (pp. 297-321). Academic Press: San Diego.
Guerry, J. D., \& Prinstein, M. J. (2010). Longitudinal prediction of adolescent nonsuicidal self-injury: Examination of a cognitive vulnerability-stress model. Journal of Clinical Child and Adolescent Psychology, 39, 77-89.

Hawker, D. S. J., \& Boulton, M. J. (2000). Twenty years' research on peer victimization and psychosocial maladjustment: A metaanalytic review of cross-sectional studies. Journal of Child Psychology and Psychiatry and Allied Disciplines, 41, 441-455.

Hay, C., \& Meldrum, R. (2010). Bullying victimization and adolescent self-harm: Testing hypotheses from general strain theory. Journal of Youth and Adolescence, 39, 446-459.

Hilt, L. M., Nock, M. K., Lloyd-Richardson, E. E., \& Prinstein, M. J. (2008). Longitudinal study of nonsuicidal self-injury among young adolescents. The Journal of Early Adolescence, 28, 455-469.

Holmbeck, G. N. (1997). Toward terminological, conceptual, and statistical clarity in the study of mediators and moderators: Examples from the child-clinical and pediatric psychology literatures. Journal of Consulting and Clinical Psychology, 4, 599-610.

Hu, L., \& Bentler, P. M. (1999). Cutoff criteria for fit indexes in covariance structure analysis: Conventional criteria versus new alternatives. Structural Equation Modeling, 6, 1-55.

Jackson, P. B. (1992). Specifying the buffering hypothesis: Support, strain and depression. Social Psychology Quarterly, 55, 363-378.

Kandel, D. B., \& Davies, M. (1986). Adult sequelae of adolescent depressive symptoms. Archives of General Psychiatry, 43, 255-262.

Kline, R. B. (2005). Principles and practices of structural equation modeling (2nd ed.). New York: Guilford Press.

Klomek, A. B., Marrocco, F., Kleinman, M., Schonfeld, I. S., \& Gould, M. (2007). Bullying, depression, and suicidality in adolescents. Journal of the American Academy of Child and Adolescent Psychiatry, 46, 40-49.

Klonsky, E. D. (2007). The functions of deliberate self-injury: A review of the evidence. Clinical Psychology Review, 27, 226-239.

Klonsky, E. D. (2009). The functions of self-injury in young adults who cut themselves: Clarifying the evidence for affect-regulation. Psychiatry Research, 27, 226-239.

Kochenderfer-Ladd, B., \& Wardrop, J. L. (2001). Chronicity and instability of children's peer victimization experiences as predictors of loneliness and social satisfaction trajectories. Child Development, 72, 134-151.

Luukkonen, A. H., Räsänen, P., Hakko, H., \& Riala, K. (2009). Bullying behavior is related to suicide attempts but not to selfmutilation among psychiatric inpatient adolescents. Psychopathology, 42, 131-138.

Muehlenkamp, J.J., Claes, L., Havertape, L., \& Plener, P.L. (2012). International prevalence of adolescent non-suicidal self-injury and deliberate self-harm. Child and Adolescent Psychiatry and Mental Health, 6:10. www.capmh.com/content/6/1/10.

Muehlenkamp, J. J., Claes, L., Peat, C., Smits, D., \& Vandereycken, W. (2011). Non-suicidal self-injury in eating disordered patients: A test of a conceptual model. Psychiatry Research, 188, 102-108.

Nansel, T. R., Overpeck, M., Pilla, R. S., Ruan, W. J., SimonsMorton, B., \& Scheidt, P. (2001). Bullying behaviors among US youth: Prevalence and association with psychosocial adjustment. Journal of the American Medical Association, 285, 2094-2210.

Noble, R. N., Sornberger, M. J., Toste, J. R., Heath, N. L., \& McLouth, R. (2011). Safety first: The role of trust and school safety in non-suicidal self-injury. McGill Journal of Education, $46,422-441$. 
Nock, M. K., \& Favazza, A. R. (2009). Non-suicidal self-injury: Origins, assessment, and treatment. Washington: American Psychological Association.

Nock, M. K., Joiner, T. E, Jr, Gordon, K., Lloyd-Richardson, E., \& Prinstein, M. J. (2006). Non-suicidal self-injury among adolescents: Diagnostic correlates and relation to suicide attempts. Psychiatry Research, 144, 65-72.

Nock, M. K., \& Prinstein, M. J. (2004). A functional approach to the assessment of self-mutilative behavior. Journal of Consulting and Clinical Psychology, 72, 885-890.

O'Conner, R. C., Rasmussen, S., Miles, J., \& Hawton, K. (2009). Self-harm in adolescents: self-report survey in schools in Scotland. The British Journal of Psychiatry, 194, 68-72.

Olweus, D. (1991). Bully/victim problems among school children: Basic facts and effects of a school based intervention program. In D. Pepler \& K. Rubin (Eds.), The development and treatment of childhood aggression. Hillsdale: Erlbaum.

Pouwelse, M., Bolman, C., Lodewijkx, H., \& Spaa, M. (2011). Gender differences and social support: mediators and moderators between peer victimization and depressive feelings. Psychology in the Schools, 48, 800-814.

Sansone, R. A., Wiederman, M. W., \& Sansone, L. A. (1998). The Self-Harm Inventory (SHI): Development of a scale for identifying self-destructive behaviours and borderline personality disorder. Journal of Clinical Psychology, 54, 973-983.

Scholte, R. H. J., Burk, W. J., \& Overbeek, G. (2013). Divergence in self- and peer-reported victimization and its association to concurrent and prospective adjustment. Journal of Youth and Adolescence, 42, 1789-1800.

Scholte, R. H. J., van Lieshout, C. F. M., \& van Aken, M. A. G. (2001). Perceived relational support in adolescence: Dimensions, configurations, and adolescent adjustment. Journal of Research on Adolescence, 11, 71-94.
Seals, D., \& Young, J. (2003). Bullying and victimization: prevalence and relationship to gender, self-esteem, and depression. Adolescence, 38, 735-747.

Smits, I., Soenens, B., Luyckx, K., Duriez, B., Berzonsky, M., \& Goossens, L. (2008). Perceived parenting dimensions and identity styles: Exploring the socialization of adolescents' processing of identity-relevant information. Journal of Adolescence, 31, 151-164.

Spriggs, A. L., Iannotti, R. J., Nansel, T. R., \& Haynie, D. L. (2007). Adolescent bullying involvement and perceived family, peer and school relations: Communalities and differences across race/ ethnicity. Journal of Adolescence Health, 41, 283-293.

Tatnell, R., Kelada, L., Hasking, P., \& Martin, G. (in press). Longitudinal analysis of adolescent NSSI: The role of intrapersonal and interpersonal factors. Journal of Abnormal Child Psychology. doi: 10.1007\%2Fs10802-013-9837-6.

Vandenberg, R. J., \& Lance, C. E. (2000). A review and synthesis of the measurement invariance literature: Suggestions, practices, and recommendations for organizational research. Organizational Research Methods, 3, 4-70.

Whitlock, J., Eckenrode, J., \& Silverman, D. (2006). Self-injurious behaviors in a college population. Pediatrics, 117, 1939-1948.

Wolff, J. C., Frazier, E. A., Esposito-Smythers, C., Becker, S. J., Burke, T. A., Cataldo, A., \& Spirito, A. (2014). Negative cognitive style and perceived social support mediate the relationship between aggression and NSSI in hospitalized adolescents. Journal of Adolescence, 37, 483-491.

Zautra, A. J. (2003). Emotions, stress and health. New York: University Press.

Zetterqvist, M., Lundh, L. G., Dahlström, Ö., \& Svedin, C. G. (2013). Prevalence and function of Non-Suicidal Self-Injury (NSSI) in a community sample of adolescents, using suggested DSM-5 criteria for a potential NSSI disorder. Journal fo Abnormal Child Psychology, 41, 759-773. 